\title{
Origin of Degradation in Si-Based All-Solid-State Li-Ion Micro-Batteries
}

Chunguang Chen, Jos F. M. Oudenhoven, Dmitri L. Danilov, Egor Vezhlev, Lu Gao, Na Li, Fokko M. Mulder, Rüdiger-A. Eichel, and Peter H. L. Notten*

Chunguang Chen, Dr. J. F. M. Oudenhoven, Dr. D. L. Danilov, Dr. Lu Gao, Prof. P. H. L. Notten

Eindhoven University of Technology, P.O. Box 513, 5600 MB Eindhoven, The Netherlands E-mail: p.h.1.notten@tue.nl

Chunguang Chen, Dr. D. L. Danilov, Prof. R. -A. Eichel, Prof. P. H. L. Notten, Forschungszentrum Jülich(IEK-9), D-52425, Jülich, Germany

Dr. J. F. M. Oudenhoven

Present address: Holst Centre/Imec, High Tech Campus 31, 5656 AE Eindhoven, The Netherlands

Dr. Egor Vezhlev

Jülich Centre for Neutron Science (JCNS), Forschungszentrum Jülich, 85748, Garching Germany

$\mathrm{Na} \mathrm{Li}$

IMDEA Materials Institute, 28906 Getafe, Madrid, Spain

Prof. F. M. Mulder

Delft University of Technology, Reactor Institute Delft, Mekelweg 15, 2629 JB Delft, The Netherlands

Prof. R.-A. Eichel

RWTH Aachen University, D-52074 Aachen, Germany

Prof. P. H. L. Notten,

University of Technology Sydney, Broadway, Sydney, NSW 2007, Australia

Keywords: all-solid-state battery; thin film; degradation; neutron depth profiling; lithium immobilization 
Like all rechargeable battery systems, conventional Li-ion batteries (LIB) inevitably suffer from capacity losses during operation. This also holds for all-solid-state LIB. In this contribution an in operando Neutron Depth Profiling (NDP) method is developed to investigate the degradation mechanism of all-solid-state, thin film $\mathrm{Si}-\mathrm{Li}_{3} \mathrm{PO}_{4}-\mathrm{LiCoO}_{2}$ batteries. Important aspects of the long-term degradation mechanisms are elucidated. It is found that the capacity losses in these thin film batteries are mainly related to lithium immobilization in the solid-state electrolyte, starting to grow at the anode/electrolyte interface during initial charging. The Li-immobilization layer in the electrolyte is induced by silicon penetration from the anode into the solid-state electrolyte and continues to grow at a lower rate during subsequent cycling. X-ray Photoelectron Spectroscopy (XPS) depth profiling and Transmission Electron Microscopy (TEM) analyses confirm the formation of such immobilization layer, which favorably functions as an ionic conductor for lithium ions. As a result of the immobilization process, the amount of free moveable lithium ions is reduced, leading to the pronounced storage capacity decay. Insights gained from this research shed interesting light on the degradation mechanisms of thin film, all-solid-state LIB and facilitate potential interfacial modifications which finally will lead to substantially improved battery performance.

\section{Introduction}

Life without portable, wireless electronics is nowadays unimaginable. The functionality of these electronic devices has increased tremendously during the last decade whereas the weight and size have decreased significantly. Wireless devices are mainly powered with on-board rechargeable batteries. The reduced size and increased functionality of present-day electronics also require small-sized batteries with high energy densities. ${ }^{[1,2]}$ Rechargeable batteries to be applied in electronics are therefore mainly based on the lithiumion chemistry, which offer the highest energy density in terms of specific energy $(\mathrm{Wh} / \mathrm{kg})$ and volumetric energy density $(\mathrm{Wh} / \mathrm{L})$. 
Conventional LIB are based on liquid electrolytes, containing Li salts dissolved in organic solvents. These electrolytes are intrinsically volatile and flammable, which requires close attention to the safety aspects of liquid-based Li-ion batteries. All-solid-state LIB, on the other hand, are generally considered more stable, providing: (i) Better safety by removing flammable liquid electrolytes; ${ }^{[3-6]}$ (ii) Wide electrochemical stability window for solid-state electrolytes, which may potentially facilitate the use of high voltage cathode materials and lead to further increase in the energy density; ${ }^{[7]}$ and (iii) Facilitate miniaturization by removing liquids and separators, allowing more flexibility for integrating microelectronic devices. ${ }^{[8]}$

Currently, the majority of commercial thin-film LIB are based on $\mathrm{LiCoO}_{2}$ as cathode, a solid electrolyte layer of $\mathrm{Li}_{3} \mathrm{PO}_{4}$ or LiPON and metallic Lithium as anode. However, metallic lithium has an extremely low melting point of $181^{\circ} \mathrm{C}$, a temperature usually lower than that applied during re-flow soldering processes widely used in the microelectronic industry. ${ }^{[1]}$ In contrast, using $\mathrm{Si}$ as anode can get rid of such potential hazards because of the high temperature tolerance. An additional advantage of Li-metal-free all-solid-state LIB is that it can operate at elevated temperatures and that the ionic conductivity of solid electrolytes increases. Si anodes have a very high theoretical storage capacity of $3579 \mathrm{mAh} \cdot \mathrm{g}^{-1}$ or 8303 $\mathrm{mAh} \cdot \mathrm{cm}^{-3}$ assuming full conversion of $\mathrm{Si}$ into $\mathrm{Li}_{15} \mathrm{Si}_{4}$. Therefore, only a very thin $\mathrm{Si}$ anode is needed, usually 10 times thinner than $\mathrm{LiCoO}_{2}$.

Apart from flat thin film battery geometries, a 3D geometry for these all-solid-state batteries has been proposed to increase the storage capacity per footprint surface area while keeping good Li-ion transportation properties. ${ }^{[1,8-10]}$ The development of all-solid-state LIB are, however, in all cases still facing quite some scientific and technical challenges. In particular, the degradation mechanisms still remain largely unexplored, even though this is one of the major research topics for large-scale, liquid-electrolyte based batteries. 
The degradation of classical liquid electrolyte LIB involves several mechanisms. One well-known mechanism is related to the formation of a Solid-Electrolyte Interphase (SEI), a film of various reaction side-products at the interface between the electrodes and the liquid electrolyte. ${ }^{[11-12]}$ It is expected that this is not occurring in solid-state batteries due to the absence of any liquid electrolyte. ${ }^{[1]}$ A second degradation mechanism of LIB is related to mechanical integrity. This seems to be more crucial for solid-state batteries as these batteries can only perform well when good contacts are established and maintained between the electrodes and the electrolyte when the electrodes swell or shrink during (de)lithiation. ${ }^{[13-15]}$ In a liquid electrolyte LIB close contact between the electrodes and electrolyte can always be secured by the free-movable liquid electrolyte. For thin-film batteries, on the other hand, (micro-)cracking and delamination may occur, which will decrease the effective contact area between the individual battery layers, and therefore may decrease the effective energy and power density. A third mechanism can be attributed to the degradation of the electrode materials. During battery cycling, and especially during over(dis)charging, side reactions may occur in the electrodes that result in electrochemically inactive species. ${ }^{[11,16]}$

Obviously, there is a need to understand the details of the degradation mechanisms involved in all-solid-state LIB. Some techniques have been applied to investigate the crystallographic changes within thin-film electrodes such as, for example, X-ray diffraction $(\mathrm{XRD})^{[17-18]}$ and Raman spectroscopy. ${ }^{[19]}$ These techniques are, however, insensitive to lithium ions and become also irrelevant when amorphous materials are involved. In situ Transmission Electron Microscopy (TEM) has also been used to investigate all-solid-state batteries, ${ }^{[20,21]}$ but this technique has a maximum resolution limit of the order of $100 \mathrm{~nm}$, not able to functionally monitor full battery stacks, and sometimes leads to chemical breakdown, and even short-circuiting because of the use of focused electron beams. Therefore a novel and flexible in operando technique is under urgent demand which is capable of determining the lithium concentration with high depth-resolution inside solid-state, thin film LIB. 
This shortcoming can be filled by Neutron Depth Profiling (NDP). NDP employs thermal neutrons (n) which react with ${ }^{6} \mathrm{Li}$, emitting alpha particles $\left({ }^{4} \mathrm{He}\right)$ and tritons $\left({ }^{3} \mathrm{H}\right)$ with well-defined initial kinetic energies of 2727 and $2055 \mathrm{keV}$, respectively, according to

$\mathrm{n}+{ }^{6} \mathrm{Li} \rightarrow{ }^{4} \mathrm{He}+{ }^{3} \mathrm{H}$

On their trajectory through various layers, the kinetic energies of ${ }^{3} \mathrm{H}$ and ${ }^{4} \mathrm{He}$ particles are reduced, depending on the material(s) passed. By detecting the energy loss of these charged particles, the depth at which these particles were initially formed can be deduced. As the number of ${ }^{6} \mathrm{Li}$ atoms subjected to this nucleus reaction is negligible with respect to the total amount of lithium, this technique can be considered non-destructive. It has been demonstrated that this method is highly sensitive for lithium because of the high neutron absorption cross section, ${ }^{[22-25]}$ and this method has therefore been successfully applied to unravel lithium depth-profiles of electrochromic lithium, thin-film devices. ${ }^{[26-28]}$ It has also been demonstrated that NDP can be applied as an ex situ measurement technique to study dismantled batteries. ${ }^{[29]}$ In situ detection was also performed to observe $\mathrm{Li}$-ion transport within individual electrodes. ${ }^{[30-32]}$ With respect to integrated all-solid-state thin-film micro-batteries, in situ NDP can also be used to investigate the processes inside complete solid-state battery stacks. ${ }^{[33]}$ In this contribution, NDP is employed as an in operando technique, to continuously monitor the degradation of thin-film micro-batteries that were cycled during a prolonged period of time. The NDP-results are supported by both XPS depth profiling and TEM analyses.

\section{Results and Discussion}

Si-based thin-film batteries with a $\mathrm{Si}$ anode $(50 \mathrm{~nm}), \mathrm{Li}_{3} \mathrm{PO}_{4}$ electrolyte $(2 \mu \mathrm{m})$ and $\mathrm{LiCoO}_{2}$ cathode $(550 \mathrm{~nm})$ were deposited. Detailed information about how the batteries were synthesized is given in the experimental section. The prepared batteries were positioned inside 
the NDP chamber and connected to a combined potentiostat/galvanostat. The NDP setup and battery configuration is shown Figure 1a. The electrochemical charge transfer reactions of the synthesized batteries can be represented by

Charge
$\mathrm{LiCoO}_{2} \stackrel{\rightarrow}{\leftarrow} \mathrm{Li}_{1-\mathrm{x}} \mathrm{CoO}_{2}+\mathrm{xLi}^{+}+\mathrm{xe}^{-}$

Discharge

$\mathrm{Li}_{\mathrm{y}} \mathrm{Si} \stackrel{\text { Charge }}{\leftarrow} \underset{\mathrm{yLi}^{+}+\mathrm{ye}^{-}+\mathrm{Si}}{ }$

Discharge

for the cathode and anode, respectively, where $\mathrm{LiCoO}_{2}$ can be cycled between $0 \leq \mathrm{x} \leq 0.5$, and Si between $0 \leq \mathrm{y} \leq 3$ in the present battery configuration, using the cathode as capacitylimiting electrode. Within these regions, both the cathode and anode can reversibly be (dis)charged upon cycling.

The as-deposited battery was then characterized by NDP. Figure $1 \mathrm{~b}$ shows the energy spectrum of an as-deposited (discharged) battery, where the full energy range is displayed, including the energy of both the $\alpha$-particles $\left({ }^{4} \mathrm{H}\right)$ and tritons $\left({ }^{3} \mathrm{H}\right)$. It is clearly visible that the higher stopping power for $\alpha$-particles results in a much wider energy range than for the tritons in this thin film battery configuration. In view of a better depth-resolution, further analyses will therefore be focused on the $\alpha$-particle energy spectra only. Subsequently, the battery was charged and discharged for two cycles with a (dis)charge current of $10 \mu \mathrm{A}(0.15 \mathrm{C}$-rate).

Figure 1c shows the difference curves of the $\alpha$-particle energy profiles, obtained by subtracting the spectrum of the pristine (discharged) battery from the first cycle fully charged (black curve) and fully discharged battery (red curve). The NDP details during charging and discharging have been continuously monitored in the difference spectra of Figure $1 \mathrm{~d}$. 
Recording each spectrum takes about 8 minutes. The cathode, electrolyte and anode regions can be clearly identified in these spectra.

Focusing for the moment on the fully charged battery only, lithium is extracted from the $\mathrm{LiCoO}_{2}$ electrode during charging (Equation 2), which obviously results in the negative cathode peak (black curve of Figure 1c) when subtracted from the pristine NDP battery signal. Obviously, $\mathrm{Li}$ is stored in the $\mathrm{Si}$ anode upon charging (Equation 3), which consequently results in a pronounced positive anode peak in the difference curve of Figure 1c. An additional interesting observation is that some extra lithium seems to be present in the electrolyte as the lithium content at the electrolyte/anode interface is clearly increased. If the battery is discharged, lithium will move in the opposite direction. Considering the NDP curve in the fully discharged state (red curve in Figure 1c), an increase of the Li-concentration in the cathode and a decrease in the anode are indeed to be expected compared to the fully charged state (black curve in Figure 1c). In case the spectrum of the fully discharged battery would behave exactly the same as that of the pristine solid-state battery, no deviation at all would become visible in the difference curve in both the cathode, electrolyte and anode regions and, consequently, a horizontal line would have been expected. However, it is already clear that in the first cycle not all Li-ions return to their original cathode positions. Some of the lithium ions remain behind in the $\mathrm{Si}$ anode and some are immobilized in the solid-state electrolyte. The observed Li-immobilization layer at the electrolyte/Si interface in the fully charged state (black curve in Figure 1c) also exists in the fully discharged state (red curve in Figure 1c). This is an interesting observation in itself as it is to be expected that immobilized lithium reduces the amount of free moveable $\mathrm{Li}$-ions and, consequently, will negatively influence the storage capacity of Si-based solid-state LIB.

In order to investigate the Li-immobilization process in more detail, about 90 NDP spectra were continuously collected during the first two (dis)charging cycles under operando conditions. The NDP results are given as a function of time in a spatial image mode in Figure 
$1 d$, for which the spectrum of pristine battery is again subtracted from each measured spectrum. The simultaneously measured battery voltage curve is shown at the left-hand side of Figure 1d. Considering the first charging process, the spectra reveal that the Liconcentration in the $\mathrm{LiCoO}_{2}$ electrode is expectedly reduced as the deep dark blue color indicates. Simultaneously, Li-ions are stored in $\mathrm{Si}$ at very high concentrations, especially at the end of the charging process, as the dark red color reveals. During discharging the reverse processes can be identified. However, the high lithium content in the cathode and zero lithium content in the Si electrode as found for the pristine battery can no longer be reached. In addition, it can be seen that a Li-containing interlayer is formed at the electrolyte/anode interface. Therefore, the formation of this Li-immobilization layer will be investigated in more detail during subsequent cycling.

For characterizing the degradation mechanism, the deposited solid-state LIB has been further cycled up to 30 cycles between 3 and $4 \mathrm{~V}$ with the same current as used for the first two cycles $(10 \mu \mathrm{A})$. The voltage curves of some selected cycles are shown as a function of time in Figure $\mathbf{2 a}$ and clearly indicate that the (dis)charge capacity is rapidly declining in these 30 cycles. The same cycles are also normalized in Figure $2 \mathrm{~b}$ with respect to the (dis)charging time, making it more distinct how the shape of the voltage curves is changing upon cycling. This facilitates to differentiate between effects that change the intrinsic processes inside the electrodes and effects that result from a decrease in the amount of mobile Li-ions. In the latter case, the shape of the normalized voltage curves remains unaltered. From Figure $2 b$ it is clear that the shape of the voltage curves changes only slightly. This is most pronounced between 3.6 and $3.9 \mathrm{~V}$. After a few cycles the sloping plateaus at $3.65 \mathrm{~V}$ disappear upon cycling. This might be due to the stabilization of the amorphous $\mathrm{Si}$ anode as the electrochemical performance in the first few cycles was always found to deviate from the subsequent cycles. $^{[34-38]}$ 
To monitor the aging effects at higher cycle numbers, the (dis)charging current after 30 cycles was increased to $20 \mu \mathrm{A}$. Using this higher current, charging and discharging was continued for several hundreds of cycles. Although the storage capacity declines at a lower rate (Figure 2c), the changes in the normalized voltage curves (Figure $2 \mathrm{~d}$ ) are only minor, indicating that the capacity loss is mainly related to the loss of mobile Li-ions. The storage capacity as a function of cycle number is shown in Figure S1. At the end of the cycle life experiment both charging and discharging conditions have been changed from constant current $(\mathrm{CC})$ into constant-current-constant-voltage $(\mathrm{CCCV}$ with $\mathrm{CC}=20 \mu \mathrm{A}$ and $\mathrm{CV}=4.0$ and 3.0 V during charging and discharging, respectively). By using this procedure, kinetic limitations are minimized and the fully available storage capacity of the battery can be utilized. The last data point in Figure S1 at cycle 364 shows that the CCCV-cycled battery hardly reveals an increased storage capacity in comparison to the constant $\mathrm{CC}$-charged cycles, suggesting that most of the lost storage capacity is indeed irreversible.

To explore the underlying degradation mechanisms, NDP spectra were continuously recorded during the entire cycling process. Figure $\mathrm{S} 2 \mathrm{a}$ and $\mathrm{b}$ show the measured energy profiles for $\alpha$ particles at the various indicated cycles as difference plots (again subtracted from the pristine battery state) in the charged and discharged state, respectively. The energy profiles show distinct differences. To view these differences in a more continuous way, all cycles are also shown in the spatial contour mode of Figure $\mathbf{3 a}$ and $\mathrm{b}$ for the charged and discharged battery state, respectively. Interestingly, the lithium level in the charged state (Figure 3a) within the delithiated cathode is more or less constant even after prolonged cycling ( 250 cycles), as the cathode region remains dark blue for all cycles. This indicates that all lithium positions inside the $\mathrm{Li}_{1-\mathrm{x}} \mathrm{CoO}_{2}$ host material are still available and that the cathode is not deteriorating upon cycling. Simultaneously, it can be seen in Figure 3a that the amount of lithium stored in the $\mathrm{Si}$ anode is significantly reduced during prolonged cycling, as the anode mapping color slowly changes from dark red into light red. Remarkably, it can also be 
seen that from the first charging cycle onwards an interlayer is formed inside the solid electrolyte starting to grow at the anode/electrolyte interface. This interlayer dramatically increases upon cycling and the electrolyte reveals a clear lithium enrichment (Figure 3 and S2).

This lithium enrichment must originate from the formation of a newly formed lithiumrich phase inside the solid-state electrolyte. Figures $3 \mathrm{a}$ and b clearly reveal that the Liimmobilization region is continuously growing during cycling. This partly explains that the anode peak is reduced upon cycling (Figure $3 \mathrm{a}$ and $\mathrm{S} 2 \mathrm{a}$ ) as the immobilized lithium is no longer available to fully charge the Si electrode. In the discharged state (Figure $3 b$ ), both the cathode and anode cannot return to the pristine state. The deviations from a horizontal line at 0 counts/s for both electrodes is again due to the fact that lithium cannot fully return to the cathode as quite some lithium remains immobilized in the solid-state electrolyte and stays behind in the anode.

In order to evaluate how much lithium is immobilized in the electrolyte and remains inside the Si electrode, the energy profiles are converted into depth profiles. Considering the lithium content and density of each individual layer, the depth profiles of the whole battery stack can be calculated from the energy difference curves. The details of these calculations are given in the Supporting Information. To the best of our knowledge, this is the first time that depth profiles for multiple Li-containing battery layers are reported. The resulting difference depth profiles as a function of cycle number are shown in Figure $3 \mathrm{c}$ and $\mathrm{d}$ for the charged and discharged states, respectively. Again it is clear that lithium becomes immobilized in the solid-state electrolyte. In addition, some lithium stays behind in the anode and is therefore no longer available to fully discharge the cathode, but this is mainly related to kinetic $\mathrm{Si}$ electrode limitations ${ }^{[39-41]}$ as NDP measurements confirm that most of the lithium residue can indeed be further extracted from the Si anode after CCCV discharging (compare the black and 
red curve in Figure S3). Strikingly, the Li-immobilization layer in the solid-state electrolyte is still present in the discharged state and is growing upon cycling.

Quantification of these degradation processes can be achieved by integration of the NDP-signal changes in the fully discharged battery states of Figure $3 \mathrm{~d}$ for the three individual electrode/electrolyte regions. The integrated, immobilized charge in the solid-state electrolyte $\left(\mathrm{Q}_{\mathrm{e}}(\mathrm{n})\right)$, anode $\left(\mathrm{Q}_{\mathrm{a}}(\mathrm{n})\right)$, and cathode $\left(\mathrm{Q}_{\mathrm{c}}(\mathrm{n})\right)$ is a function of the cycle number (n) and can be represented by

$\mathrm{Q}_{\mathrm{i}}(\mathrm{n})=\frac{\mathrm{A}_{\mathrm{i}}(\mathrm{n})}{\mathrm{A}_{\mathrm{c}}(1)} \mathrm{Q}_{\mathrm{c}}(1)$

where $\mathrm{i}$ is e, a and c accordingly. The integrated peak areas of the electrolyte $\left(A_{e}(n)\right)$, anode $\left(A_{a}(n)\right)$ and cathode $\left(A_{c}(n)\right)$ in the discharged battery state during cycling are related to the integrated cathode peak area in the charged battery state in the first cycle $\left(\mathrm{A}_{c}(1)\right)$ and the electrochemical charge introduced during the first charging $\left(\mathrm{Q}_{c}(1)\right)$. The results for the electrolyte and the Si electrode are shown by the red and black curve in Figure 3e, respectively. The amount of immobilized lithium in the electrolyte region is increasing (red curve). The amount of lithium staying behind in the Si electrode is more or less constant upon cycling (black curve). As this feature has a kinetic origin it is, formally speaking, not related to a battery ageing process. The summation of these two lines is represented by the open symbols in Figure $3 \mathrm{e}$ and represents the total loss of lithium upon cycling. The total battery capacity loss $\left(\mathrm{Q}_{\mathrm{c}}(\mathrm{n})\right)$, obtained by integration of cathode peak area, is also shown in Figure $3 \mathrm{e}$ (gray curve) and the result matches very well with the summation of the two Li-ion loss sources (open symbols). The as-determined integrated NDP capacity losses also match very well with the storage capacity decay curve shown in the inset of Figure S1, implying fast 
degradation during the initial 30 cycles followed by a more slow degradation process at higher cycle numbers.

It is obvious that due to strict electro-neutrality rules the electrolyte cannot simply contain more Li-ions than dictated by the $\mathrm{Li}_{3} \mathrm{PO}_{4}$ stoichiometry. The main question then arises in what new phase lithium has been immobilized. A possible explanation is that the formation of a new phase is induced by Si migration from the anode into the electrolyte. Silicon is wellknown as a highly reactive chemical element, which can easily form silicide compounds in contact with other materials. ${ }^{[22-45]}$ It is therefore proposed that a lithium-silicon-phosphate is formed. These multi-element phosphates have been reported to function as an excellent solidstate electrolyte and do, therefore, not impair basic solid-state LIB operation. ${ }^{[3,46-49]}$ The formation of this new phase is, however, at the expense of consuming both lithium and silicon, and this therefore strongly reduces the storage capacity of Si-based solid-state batteries upon cycling.

Lithiation of $\mathrm{Si}$ in the first cycle is already essential in initiating the degradation process. It is therefore crucial to unveil the changes of the $\mathrm{Si} / \mathrm{Li}_{3} \mathrm{PO}_{4}$ interface during this process, which is of key significance in understanding the degradation mechanism(s). To address this issue further, X-ray Photoelectron Spectroscopy (XPS) depth profiling analyses have been carried out to investigate the evolution of the $\mathrm{Si} / \mathrm{Li}_{3} \mathrm{PO}_{4}$ interface. Figure 4a shows the $\mathrm{Si} 2 \mathrm{p}$ and $\mathrm{Li} 1$ s spectra of a pristine, as-deposited, battery. Clearly, both the $\mathrm{Si} 2 \mathrm{p}$ and $\mathrm{Li}$ 1s depth profiles show that the transition from the $\mathrm{Si}$ anode to the $\mathrm{Li}_{3} \mathrm{PO}_{4}$ electrolyte is very sharp and that no interfacial layer is present in the pristine state. However, after the first charging (lithiation), the transition between $\mathrm{Si}$ and $\mathrm{Li}_{3} \mathrm{PO}_{4}$ is no longer sharp. Si migrates into the solid-state electrolyte (Figure 4b), resulting in the formation of the interfacial layer. The $\mathrm{Si}$ signal gradually decreases in the bulk of $\mathrm{Li}_{3} \mathrm{PO}_{4}$. The reflections, denoted as $\mathrm{Si}-\mathrm{O}-\mathrm{P},{ }^{[50-51]}$ are also a clear indication of the formation of such a new phase. Another interesting observation for the Si electrode is the significant increase of the electrode thickness up to about $150 \mathrm{~nm}$ 
and the accompanying decrease in XPS signal intensity (compare Figure $4 a$ and $b$ ). This is caused by volume expansion upon Li-ion insertion, inducing a much less compact morphology.

In close connection to the $\mathrm{Si}$ migration into the electrolyte, lithium is also trapped in the interfacial electrolyte region as the $\mathrm{Li}$-enriched signal near the $\mathrm{Si} / \mathrm{Li}_{3} \mathrm{PO}_{4}$ interface shows in the Li 1s spectra in Figure 4b. This observation agrees quite well with the NDP observations shown in Figure 3c. The immobilized Li-ions can unfortunately not be remobilized anymore, as has been concluded from Figure $3 \mathrm{~d}$. This is confirmed by the XPS depth profile of the first discharge cycle (Figure 4c), which shows that both the Si-O-P reflections and the $\mathrm{Li}$-enrichment signals are still present in the $\mathrm{Li}_{3} \mathrm{PO}_{4}$ electrolyte. Owing to the $\mathrm{Li}$ extraction, the $\mathrm{Si}$ anode signal increases again towards higher intensities, indicating a more compact structure compared to lithiated Si. However, the original thickness and XPS intensity of the Si anode (compare Figure 4a and c) is not fully recovered, which is due to the incomplete delithiation of $\mathrm{Si}$, originating from the kinetic limitations. The front view of one $\mathrm{Si}$ $2 p$ XPS spectrum corresponding to where the Li-immobilization layer has been formed is shown in Figure S4 in detail.

TEM observations also confirm the growth of such interlayer. The TEM image of the pristine $\mathrm{Si} / \mathrm{Li}_{3} \mathrm{PO}_{4}$ interface is shown in Figure $4 \mathrm{~d}$ (left panel) and clearly shows a sharp electrode/electrolyte interface. Elemental energy dispersive X-ray spectroscopy (EDX) mapping for $\mathrm{Si}$ and $\mathrm{P}$ also indicate that there is no mixing between the two layers (second and third panel in Figure 4d, respectively). Figure 4e shows the $\mathrm{Si} / \mathrm{Li}_{3} \mathrm{PO}_{4}$ interface after the first charging has become much more diffuse: a clear interfacial region (left panel in Figure 4e) is formed between $\mathrm{Si}$ and $\mathrm{Li}_{3} \mathrm{PO}_{4}$, which is confirmed by the elemental EDX results for $\mathrm{Si}$ and $\mathrm{P}$ (second and third panels in Figure 4e, respectively). In contrast to the sharp EDX line scan shown in the $4^{\text {th }}$ panel of Figure $4 d$, a much more diffuse Si-gradient is found in the line scan of Figure 4e, starting from the anode penetrating into the solid-state electrolyte. This again 
confirms that the origin of the interfacial layer is related to Si migration into the solid-state electrolyte. As expected the interfacial layer is conserved in the discharged state as Figure $4 \mathrm{f}$ reveals.

Since three electrochemical procedures (charging, resting and discharging) were applied during cycling, it is interesting to investigate the impact of these stages on the growth process of the Li-immobilization interlayer. This could give a crucial answer of how and when $\mathrm{Si}$ migrates into the $\mathrm{Li}_{3} \mathrm{PO}_{4}$ solid-state electrolyte. The voltage curve of Figure $5 \mathrm{a}$ indicates the three periods of charging from $\mathrm{A}$ to $\mathrm{B}$, resting from $\mathrm{B}$ to $\mathrm{C}$ and discharging from C to D. The first hypothesis would simply be that the steep concentration gradient of Si at the $\mathrm{Si} / \mathrm{Li}_{3} \mathrm{PO}_{4}$ interface is driving $\mathrm{Si}$ into the electrolyte. Against this hypothesis is the fact the pristine battery was stored for three weeks under open-circuit conditions before the in operando experiments were initiated and no mixing was found with NDP, XPS and TEM even after such a long resting period. To further investigate the impact of resting between charging and discharging, NDP energy spectra were continuously recorded for a 21 hour resting period after the first charging. Figure $5 \mathrm{c}$ shows the development of the operando spatial image during the entire relaxation period. This result clearly shows that the Liimmobilization interlayer is not expanding further during the extended relaxation time measured between B and C in Figure 5a. Therefore we can conclude that the formation of the interface layer is not concentration driven by $\mathrm{Si}$ and that $\mathrm{Si}$ migration into the solid-state electrolyte is induced by the charging and/or discharging current.

In order to analyze Si-migration under current flowing conditions, spatial incremental images are considered just before charging (at position A), at the end of charging (B), just before discharging (C) and at the end of discharging (D). The incremental spatial images for charging (B-A) and discharging (D-C) are shown for the first 30 cycles in Figure $5 \mathrm{~b}$ and d, respectively. Focusing on the electrolyte region in the energy range of 800 to $1500 \mathrm{keV}$ during charging (Figure 5b), it is apparent that the Li-concentration differences between the 
fully charged and fully discharged state (B-A) in the solid-state electrolyte is quite inhomogeneous but locally increases to relatively high values as the red regions near the $\mathrm{Si} / \mathrm{Li}_{3} \mathrm{PO}_{4}$ interface indicate. In contrast, during discharging (Figure 5d), the differences between the fully discharged and fully charged state $(\mathrm{D}-\mathrm{C})$ is negligible, implying that the Liimmobilization layer is more or less stable and is not growing during the discharging periods. The findings shown in Figure 5b-d clearly demonstrate that the Li-immobilization interlayer is mainly developed during the charging process.

The fact that Li-immobilization in the solid-state electrolyte only takes place under charging conditions when Li-ions are transported from the cathode via the electrolyte to the $\mathrm{Si}$ anode is highly relevant. It is well-known that the lithiation process of Si induces very large volume expansions inside the anode. Especially at the $\mathrm{Si} / \mathrm{Li}_{3} \mathrm{PO}_{4}$ interface this may create an extreme distortion of the host material through which the Si mobility can be drastically increased, facilitating Si migrating into the electrolyte. A second aspect which may play a role is that $\mathrm{Li}$-ions are hopping inside the solid-state electrolyte via a vacancy hopping mechanism. ${ }^{[52-53]} \mathrm{Li}$-ions have a radius of $0.128 \mathrm{~nm}$, which is larger than that of $\mathrm{Si}(0.111$ $\mathrm{nm})$. The vacancies induced in the solid-state electrolyte under current flowing conditions can therefore easily host the highly mobile, migrated Si atoms. Simultaneously more Li-ions are immobilized in the electrolyte fulfilling the electroneutrality rule for this new chemical compound. Despite the fact that the vacancy hopping mechanism and Si volume changes during discharging are in the opposite direction, the migrated $\mathrm{Si}$ remains in the $\mathrm{Li}$ immobilization interlayer, likely due to the fact that this compound is thermodynamically rather stable in comparison to that of pure $\mathrm{Li}_{3} \mathrm{PO}_{4}$. This might be an interesting observation, which will be further explored in future work. The development of the degradation process is schematically summarized in Figure 5e for the pristine, charging, fully charged, discharging and fully discharged state. 
The degradation observed in the present study seems to deviate from our previously reported half-cell experiments, where a $\mathrm{Li}_{3} \mathrm{PO}_{4}$-protected $\mathrm{Si}$ thin film anode of $50 \mathrm{~nm}$ thickness revealed a much better cycle-life. ${ }^{[38]}$ However, this can be fully explained by the battery configuration used in the present work where $\mathrm{Li}_{3} \mathrm{PO}_{4}$ layer thickness of $2 \mu \mathrm{m}$ is used, while in our previous study a $\mathrm{Li}_{3} \mathrm{PO}_{4}$ layer thickness of only $200 \mathrm{~nm}$ was used. ${ }^{[38]}$ Although a very stable cycle-life performance was observed in the latter case, a relatively large capacity drop was also present during initial activation. ${ }^{[38]}$ This capacity loss can again be attributed to the formation of an interfacial $\mathrm{Si}$ - and $\mathrm{Li}$-immobilization layer. However, as only $200 \mathrm{~nm}$ of $\mathrm{Li}_{3} \mathrm{PO}_{4}$ was used in that specific case, $\mathrm{Li}_{3} \mathrm{PO}_{4}$ becomes already fully saturated with $\mathrm{Si}$ in the initial stages of cycling and further immobilization of $\mathrm{Si}$ and $\mathrm{Li}$ does no longer occur. Moreover, these half-cell measurements ${ }^{[38]}$ were performed against a metallic lithium counter electrode, which offers a large excess of lithium. Capacity losses due to the immobilization of lithium in the solid electrolyte can therefore not be monitored. In the full solid-state batteries considered in the present paper, on the other hand, no excess of lithium is available. The immobilization of lithium in an interfacial layer has therefore a clear and direct impact on the cycle-life performance of all-solid-state batteries. Consequently, it can be expected that the occurring interfaces are common for all Si-based solid-state LIB, although the impact on the cycle life will be strongly dependent on the battery configuration. From the present results it can be concluded that developing stable interfaces for Si-based all-solid-state Li-ion batteries is therefore essential to create long cycle-life performance.

\section{Conclusions}

Neutron Depth Profiling was shown to be a very powerful method to in operando investigate the degradation mechanism of all-solid-state $\mathrm{Li}$-ion batteries. It was found by NDP that degradation of Si-based all-solid-state batteries originates from the immobilization of lithium inside the electrolyte. A detailed degradation mechanism has been unveiled by combining NDP with supportive XPS and TEM measurements. It has been demonstrated that 
Li-immobilization is induced by Si-migration into the solid-state electrolyte under current flowing conditions. The Si-incorporated solid-state electrolyte is chemically modified in such a way that more lithium can be included in the as-formed interfacial layer. The formation of such interfacial electrolyte layer unfortunately goes at the expense of the storage capacity of the thin film batteries upon cycling as reversible Li-ions are immobilized in this new phase and can no longer be extracted during normal battery operation.

Information gained from this work can, however, open up new prospect of analyzing the interface characteristics of various all-solid-state LIB in much more detail under in operando conditions. Further work is currently ongoing to introduce specific protective layers between $\mathrm{Si}$ and $\mathrm{Li}_{3} \mathrm{PO}_{4}$, which function as barrier for Si-migration, thereby preventing $\mathrm{Li}$ immobilization and, consequently, effectively improving the cycling performance of all-solidstate Li-ion micro-batteries. We will report on this successful strategy in forthcoming paper(s). Another potential strategy is to employ the optimal and thermodynamically favorable lithium-silicon-phosphate composition as solid-state electrolyte in all-solid-state batteries, in which case Si migration into the electrolyte will be prevented as it is already saturated with Si.

\section{Experimental Section}

\section{Batteries manufacturing process}

All-solid-state, thin-film Li-ion batteries were fabricated by sputtering and e-beam evaporation methods. The batteries have the following configuration (see inset of Figure 1a): $200 \mathrm{~nm}$ sputtered Pt current collector, $550 \mathrm{~nm}$ sputtered $\mathrm{LiCoO}_{2}$ cathode, $2.0 \mu \mathrm{m}$ sputtered $\mathrm{Li}_{3} \mathrm{PO}_{4}$ solid-state electrolyte, $50 \mathrm{~nm}$ e-beam evaporated $\mathrm{Si}$ anode, $150 \mathrm{~nm}$ e-beam evaporated copper current collector and the batteries were finally sealed with packaging coatings. Silicon was chosen as anode material, because it has a potentially very high gravimetrical storage capacity, ${ }^{[1,34]}$ and only very thin anode films are therefore required in comparison to the 
cathode electrode thickness. Such thin film Si electrodes were reported to be mechanically stable upon repeatedly (dis)charging. ${ }^{[1,34]}$ It is worthwhile to note that the aim of this experiment is to study ageing, in particular to study the migration of $\mathrm{Si}$. Therefore, the design of the test sample should (i) make possible migration of a considerable amount of Si into the $\mathrm{Li}_{3} \mathrm{PO}_{4}$, (ii) make the formation of the Li-enriched interlayer visible by NDP resolution. To comply with these requirements the $\mathrm{Li}_{3} \mathrm{PO}_{4}$ layer with a thickness 2 um was used. Moreover, the total capacity of the test cell is not very high and the Si layer is only $50 \mathrm{~nm}$ thick. Therefore, the effect of Si migration and subsequent Li-immobilization upon battery degradation becomes clearly visible.

\section{Electrochemical analyses}

The as-produced solid-state batteries are connected to a Keithley 2400 sourcemeter to perform the electrochemical cycling, controlled by an in-house developed Borland Delphi program. The batteries were cycled at a constant current (dis)charging procedure for 350 cycles and then followed with a CCCV (dis)charging method. The cutoff voltages were set at 3.00 and $4.00 \mathrm{~V}$ for charging and discharging at 10 or $20 \mu \mathrm{A}$, respectively. After every constant current (dis)charge step the battery was left to rest for 1 hour. Occasionally the influence of a longer resting period was investigated as indicated in the text.

\section{In operando NDP analyses}

The NDP experiments were carried out with a low energy thermal neutron beam line at the Reactor Institute Delft (The Netherlands). The NDP analyzing system is schematically shown in Figure 1a. The NDP set-up consisted of a vacuum chamber that contained a neutron beam monitor, a charged particle detector, a neutron beam stop and a frame to mount the batteries. The thin film batteries were mounted at an angle of 30 degrees to the incoming neutron beam, facing towards the detector. On top of the batteries, a neutron absorbing aperture was placed 
to delimit a well-defined area for the NDP analyses. Data collection of a single NDP spectrum takes approximately 8 minutes, which is relatively short with respect to the (dis)charging time of 6 hour and 3 hour for a $10 \mu \mathrm{A}$ and $20 \mu \mathrm{A}$ (dis)charge current followed by a 1 hour resting period. The results obtained directly from the NDP analyzer are energy spectra profiles. Based on energy losses the triton or alpha formation depths can be deduced when these particles reach the detector, assuming that detailed information of the battery morphology/chemistry are known. A detailed description how the energy profiles have been transformed into depth profiles is described in detail in the Supporting Information. 


\section{Characterization of the $\mathrm{Si} / \mathrm{Li}_{3} \mathrm{PO}_{4}$ interface}

XPS depth profiles were obtained with a ThermoScientific K-Alpha instrument equipped with a monochromatic $\mathrm{X}$-ray source $(\mathrm{Al} \mathrm{K \alpha}=1486.6 \mathrm{eV})$. The thicknesses, as measured by SEM, were compared with the number of sputter cycles that took place before the $\mathrm{Cu}$ surface was reached.

Samples for TEM observations were prepared, using focused ion beam (FIB) milling to thin down the specimen to less than $100 \mathrm{~nm}$. The anode-electrolyte interfaces were investigated using a TEM (FEI TalosTM F200). Elemental mapping to investigate the crosssection of the interfaces were conducted by an EDX spectrometer (Rayny EDX-800HS, Shimadzu). The samples were transferred under Ar from a glove box (Unilab PRO, MBraun) for the XPS, FIB and TEM analyses.

\section{Supporting Information}

Supporting Information is available from the authors.

\section{Acknowledgements}

C. C. acknowledges the Chinese Scholarship Council (Grant No. 201506020080) for partially financial support of this work. D.L.D. appreciates the financial support from the Horizon 2020 program of the European Union under the DEMOBASE project (Grant No. 769900). The authors gratefully thank Mr. A. Weber for the technical support in batteries deposition and Mr. F. Labohm for his support in setting up the in operando NDP experiments.

\section{References}

[1] P. H. L. Notten, F. Roozeboom, R. A. H. Niessen, L. Baggetto, Adv. Mater. 2007, 19, 4564.

[2] M. Armand and J. -M. Tarascon, Nature 2008, 451, 652.

[3] J. B. Bates, N. J. Dudney, G. R. Gruzalski, R. A. Zuhr, A. Choudhury, C. F. Luck, J. D. Robertson, Solid State Ionics 1992, 53-56, 647. 
[4] J. B. Bates, N. J. Dudney, B. Neudecker, A. Ueda, C. D. Evans, Solid State Ionics 2000, $135,33$.

[5] B. J. Neudecker, N. J. Dudney, J. B. Bates, J. Electrochem. Soc. 2000, 147, 517.

[6] J. Janek, W. G. Zeier, Nat. Energy 2016, 1, 1.

[7] A. C. Luntz, J. Voss, K. Reuter, J. Phys. Chem. Lett. 2015, 6, 4599.

[8] J. F. M. Oudenhoven, L. Baggetto, P. H. L. Notten, Adv. Energy Mater. 2011, 1, 10.

[9] J. W. Long, B. Dunn, D. R. Rolison, H. S. White, Chem. Rev. 2004, 104, 4463.

[10] M. Létiche, E. Eustache, J. Freixas, A. Demortière, V. De Andrade, L. Morgenroth, P. Tilmant, F. Vaurette, D. Troadec, P. Roussel, T. Brousse, C. Lethien, Adv. Energy Mater. 2017, 7, 1601402.

[11] J. Vetter, P. Novák, M. R. Wagner, C. Veit, K. -C. Möller, J. O. Besenhard, M. Winter, M. Wohlfahrt-Mehrens, C. Vogler, A. Hammouche, J. Power Sources 2005, 147, 269.

[12] P. Verma, P. Maire, P. Novák, Electrochimica Acta 2010, 55, 6332.

[13] B. B. Wu, S. Y. Wang, W. J. Evans IV, D. Z. Deng, J. H. Yang, J. Xiao, J. Mater. Chem. A 2016, 4, 15266.

[14] Y. Z. Zhu, X. F. He, Y. F. Mo, J. Mater. Chem. A 2016, 4, 3253.

[15] A. Brazier, L. Dupont, L. Dantras-Laffont, N. Kuwata, J. Kawamura, J. -M. Tarascon, Chem. Mater. 2008, 20, 2352.

[16] M. Wohlfahrt-Mehrens, C. Vogler, J. Garche, J. Power Sources 2004, 127, 58.

[17] L. Baggetto. P. H. L. Notten, J. Electrochem. Soc. 2009, 156, A169.

[18] T. D. Hatchard, J. R. Dahn, J. Electrochem. Soc. 2004, 151, A838.

[19] K. Dokko, M. Mohamedi, N. Anzue, T. Itoh, I. Uchida, J. Mater. Chem. 2002, 12, 3688.

[20] Z. Y. Wang, D. Santhanagopalan, W. Zhang, F. Wang, H. L. L. Xin, K. He, J. C. Li, N. Dudney, Y. S. Meng, Nano Lett. 2016, 16, 3760. 
[21] D. Ruzmetov, V. P. Oleshko, P. M. Haney, H. J. Lezec, K. Karki, K. H. Baloch, A. K. Agrawal, A. V. Davydov, S. Krylyuk, Y. Liu, J. Y. Huang, M. Tanase, J, Cumings, A. A. Talin, Nano Lett. 2012, 12, 505.

[22] G. P. Lamaze, H. H. Chen-Mayer, D. A. Becker, F. Vereda, R. B. Goldner, T. Haas, P. Zerigian, J. Power Sources 2003, 119-121, 680.

[23] L. Salavcova, J. Spirkova, F. Ondracek, A. Mackova, J. Vacik, U. Kreissig, F. Eichhorn, R. Groetzschel, Optical Mater. 2007, 29, 913.

[24] S. M. Whitney, S. R. Biegalski, Y. H. Huang, J. B. Goodenough, J. Electrochem. Soc. 2009, 156, A886.

[25] S. M. Whitney, S. R. F. Biegalski, G. Downing, J. Radioanal. Nucl. Chem. 2009, 282, 173. [26] L. H. M. Krings, Y. Tamminga, J. van Berkum, F. Labohm, A. van Veen, W.M. Arnoldbik, J. Vac. Sci. Technol. A 1999, 17, 198.

[27] G. P. Lamaze, H. Chen-Mayer, M. Badding, M. L. Laby, Surf. Interface Anal. 1999, 27, 644.

[28] G. P. Lamaze, H. H. Chen-Mayer, A. Gerouki, R. B. Goldner, Surf. Interface Anal. 2000, 29,638 .

[29] S. C. Nagpure, R. G. Downing, B. Bhushan, S. S. Babu, L. Cao, Electrochim. Acta 2011, $53,4735$.

[30] D. X. Liu, J. H. Wang, K. Pan, J. Qiu, M. Canova, L. R. Cao, A. C. Co, Angew. Chem. Int. Ed. 2014, 53, 9498 .

[31] X. Y. Zhang, T. W. Verhallen, F. Labohm, M. Wagemaker, Adv. Energy Mater. 2015, 5, 1500498.

[32] D. X. Liu, A. C. Co, J. Am. Chem. Soc. 2016, 138, 231.

[33] J. F. M. Oudenhoven, F. Labohm, M. Mulder, R. A. H. Niessen, F. M. Mulder, P. H. L. Notten, Adv. Mater. 2011, 23, 4103. 
[34] L. Baggetto, R. A. H. Niessen, F. Roozeboom, P. H. L. Notten, Adv. Funct. Mater. 2008, $18,1057$.

[35] V. Baranchugov, E. Markevich, E. Pollak, G. Salitra, D. Aurbach, Electrochem. Commun. 2007, 9, 796.

[36] H. Jung, M. Park, Y-G. Yoon, G-B. Kim, S-K. Joo, J. Power Sources 2003, 115, 346.

[37] S. Bourderau, T. Brouse, D. M. Schleich, J. Power Sources 1999, 81-82, 233.

[38] J. Xie, J. F. M. Oudenhoven, P-P. R. M. L. Harks, D. J. Li, P. H. L. Notten, J Electrochem Soc, 2015, 162, A249.

[39] H. Li, X. J. Huang, L. Q. Chen, G. W. Zhou, Z. Zhang, D. P. Yu, Y. J. Mo, N. Pei, Solid State Ionics 2000, 135, 181.

[40] U. Wahl, H. Hofsäss, S. G. Jahn, S. Winter, E. Recknagel, Appl. Phys. Lett. 1993, 62, 684.

[41] U. Kasavajjula, C. S. Wang, A. J. Appleby, J. Power Sources 2007, 163, 1003.

[42] M. Bohner, Biomaterials, 2009, 30, 6403.

[43] R. D. Thompson, B. Y. Tsaur, K. N. Tu, Appl. Phys. Lett. 1981, 38, 535.

[44] S. K. Zheng, J. W. Feng, J. A. Diverdi, G. E. Maciel, Inorg. Chem. 2006, 45, 6073.

[45] D. -S. Lin, T. -S. Ku, T. -J. Sheu, Surface Science 1999, 424, 7.

[46] Y. M. Zhu, W. F. Chu, W. Weppner, Chinese SCI Bull 2009, 54, 1334.

[47] K. Kanehori, K. Matsumoto, K. Miyauchi, T. Kudo, Solid State Ionics 1983, 9/10, 1445.

[48] Y. R. Su, J. Falgenhauer. T. Leichtwei $\beta$, M. Geiß, C. Lupó, A. Polity, S. Q. Zhou, J. Obel,

D. Schlettwein, J. Janek, B. K. Meyer, Phys. Status Solidi B 2017, 254, 1600088.

[49] M. Aslam, X. Y, Kong, Solid State Ionics 2016, 293, 72.

[50] C. L. Yang, W. J. Weng, P. Y. Du, G. Sheng, G. R. Han, Key Eng. Mater. 2007, 330-332, 765.

[51] J. G. Liao, X. J. Wang, Y. Zuo, L. Zhang, J. Q. Wen, Y. B. Li, Journal of Inorganic Materials 2008, 23, 145. 
[52] D. Danilov, R. A. H. Niessen, P. H. L. Notten, J Electrochem Soc, 2011, 158, A215.

[53] Y. A. Du, N. A. W. Holzwarth, J Electrochem Soc, 2007, 154, A999.

[54] D. Santhanagopalan, D. Qian, T. McGilvray, Z. Y. Wang, F. Wang, F. Camino, J.

Graetz, N. Dudney, Y. S. Meng, J. Phys. Chem. Lett. 2014, 5, 298.

[55] F. L. Cras, B. Pecquenard, V. Dubois, V-P. Phan, D. Guy-Bouyssou, Adv. Energy Mater. 2015, $1,1501061$. 
a

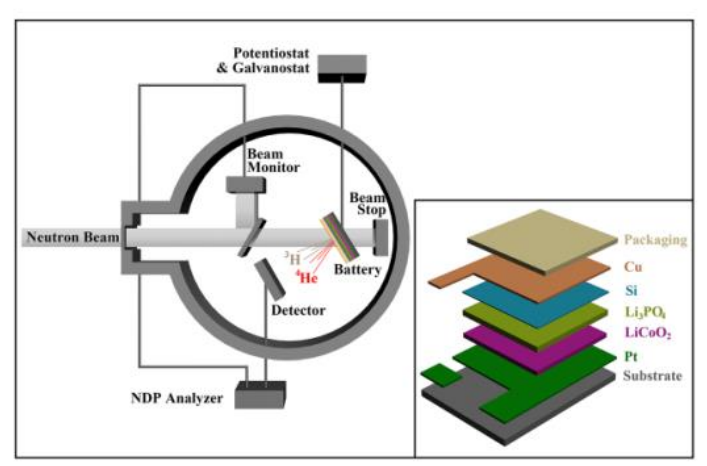

C

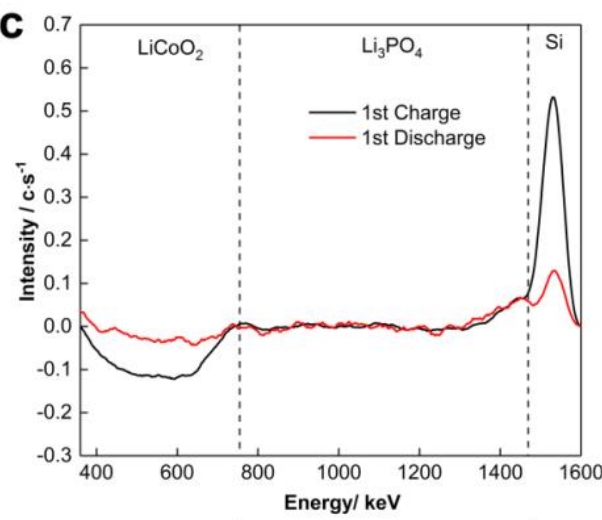

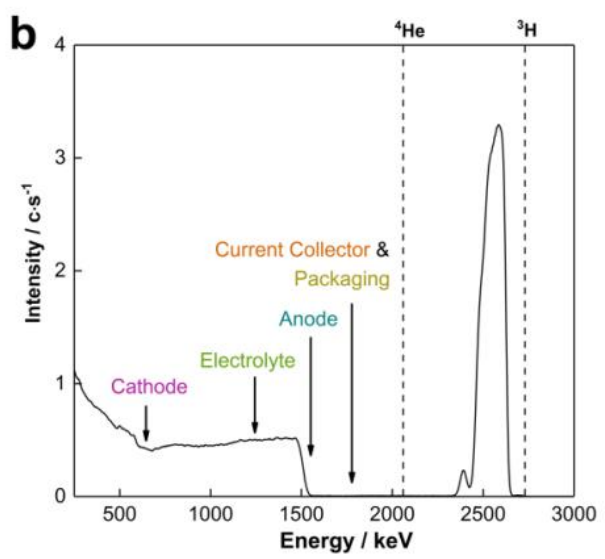

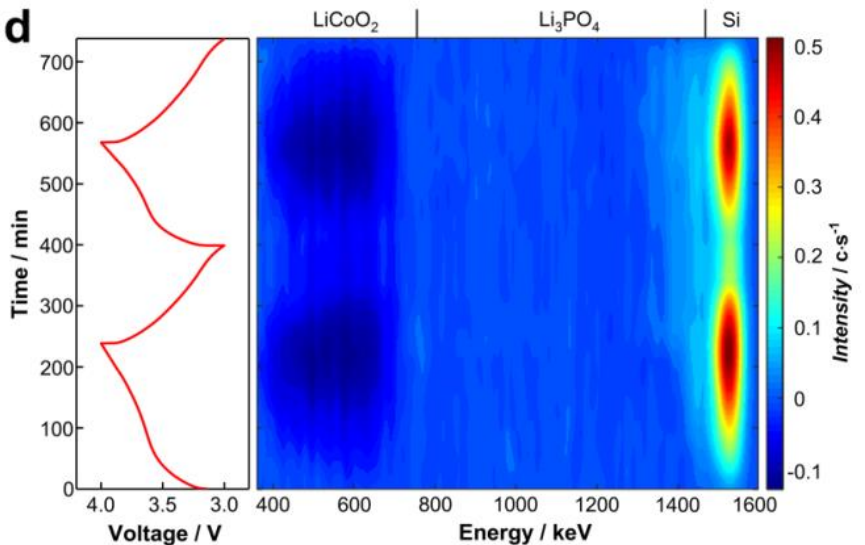

Figure 1. (a) Schematic representation of the NDP set-up, where the battery is mounted at an angle of 30 degrees with respect to the incoming neutron beam. An electric feed-through facilitates the connections of the battery to a potentiostat or galvanostat, in this case a Keithley 2400 sourcemeter. The inset shows the layer-by-layer $\mathrm{Si}-\mathrm{Li}_{3} \mathrm{PO}_{4}-\mathrm{LiCoO}_{2}$ thin-film battery configuration. (b) NDP spectrum of an as-deposited, pristine, all-solid-state Li-ion battery. As reference, the formation energies of the triton and $\alpha$-particles are represented by the dashed vertical lines. (c) Difference energy spectra at the charged (black) and discharged (red) state of the first (dis)charging cycle for which the signal of the pristine (discharged) battery has been subtracted after background noise removal. (d) Development of the in operando spatial difference energy spectra during the first two charging and discharging cycles with the measured battery voltage curves at the left-hand side. 

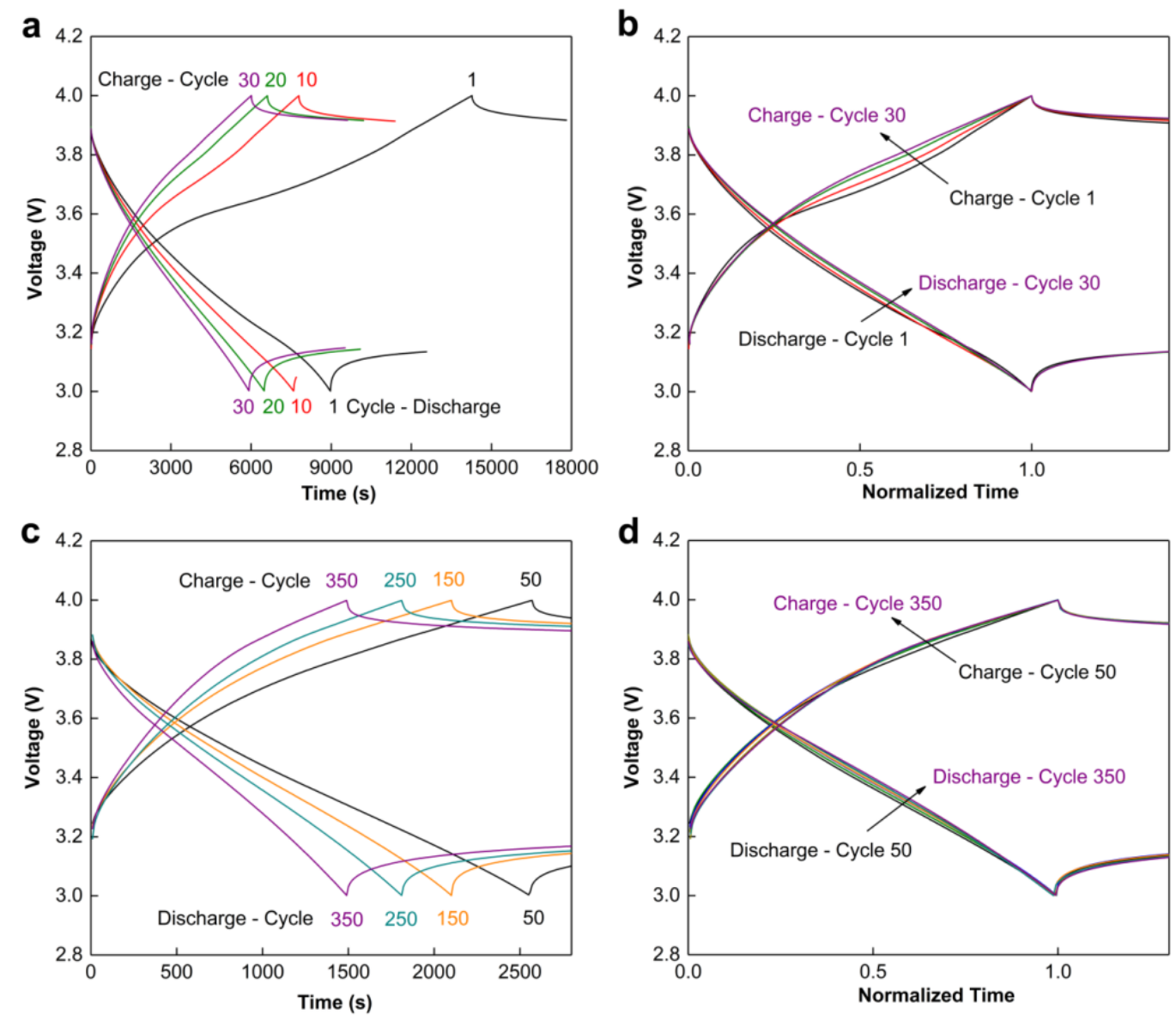

Figure 2. Voltage charging and discharging curves of a Si- $\mathrm{Li}_{3} \mathrm{PO}_{4}-\mathrm{LiCoO}_{2}$ thin-film battery during the first indicated 30 cycles with a current of $10 \mu \mathrm{A}$ as a function of time (a) and normalized with respect to the charging time (b). The subsequent cycles are (dis)charged with a current of $20 \mu \mathrm{A}(\mathrm{c})$ and (d). 

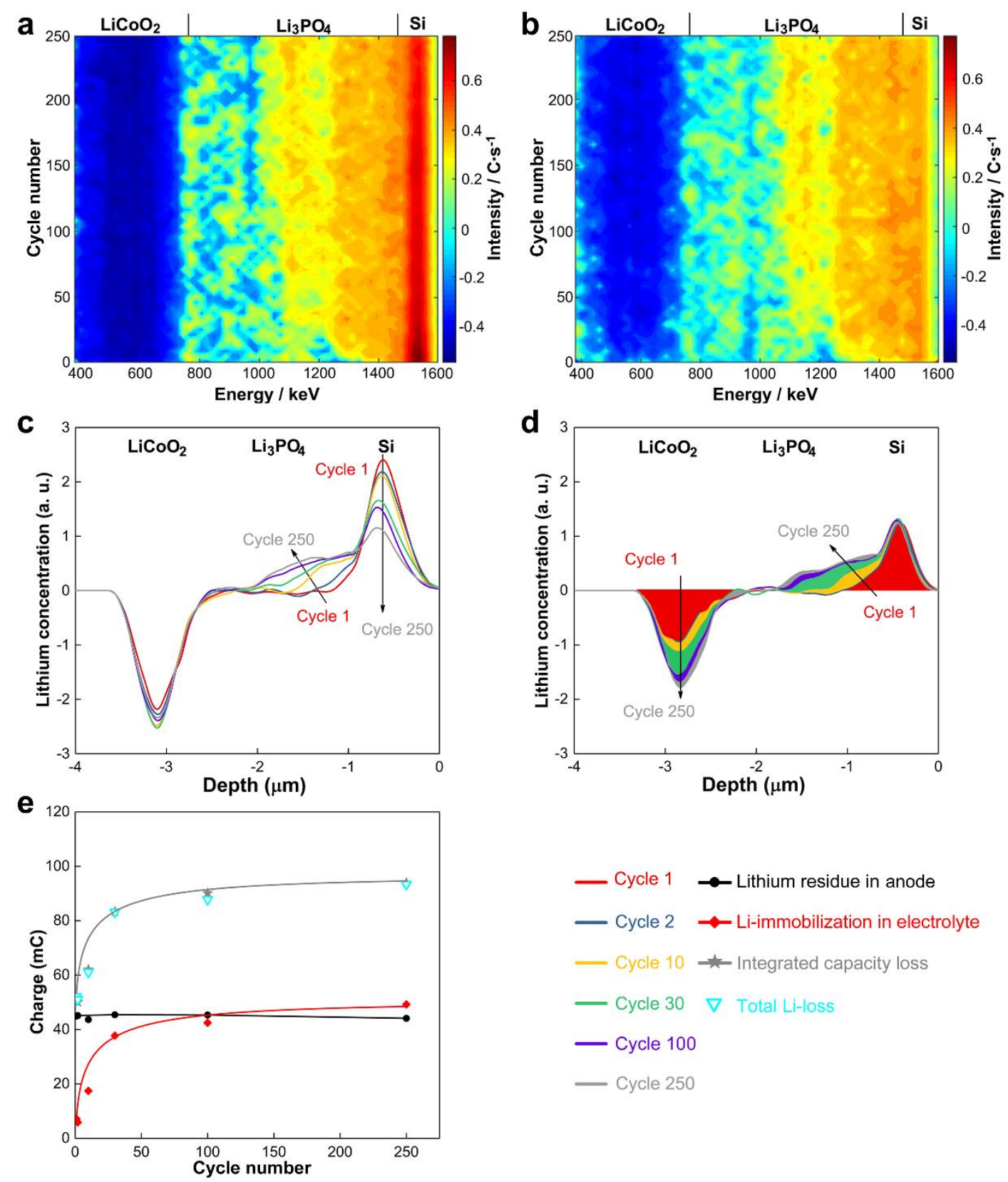

Figure 3. Development of the measured NDP spectra as a function of energy of a fully charged (a) and discharged (b) battery upon cycling up to 250 cycles. As-calculated depth profiles of the normalized lithium concentration at the charged (c) and discharged (d) state in the $1^{\text {st }}($ red $), 2^{\text {nd }}$ (blue), $10^{\text {th }}$ (yellow), 30 ${ }^{\text {th }}$ (green), 100 ${ }^{\text {th }}$ (purple) and $250^{\text {th }}$ (grey) cycle for which the signal of the pristine (discharged) battery has been subtracted. (e) Integrated NDP curves of the discharged battery shown in Figure $3 \mathrm{~d}$ as a function of cycle number. The red curve represents the integrated capacity loss due to lithium immobilization in the solid-state electrolyte $\left(\mathrm{Q}_{\mathrm{e}}(\mathrm{n})\right)$ and the black curve shows the integrated lithium residue in the Si anode $\left(\mathrm{Q}_{\mathrm{a}}(\mathrm{n})\right)$. The open cyan symbols show the summation of the integrated signals of the electrolyte and $\mathrm{Si}$ anode, which agrees very well with the grey curve, representing the integrated capacity reduction of the cathode $\left(\mathrm{Q}_{\mathrm{c}}(\mathrm{n})\right)$. 

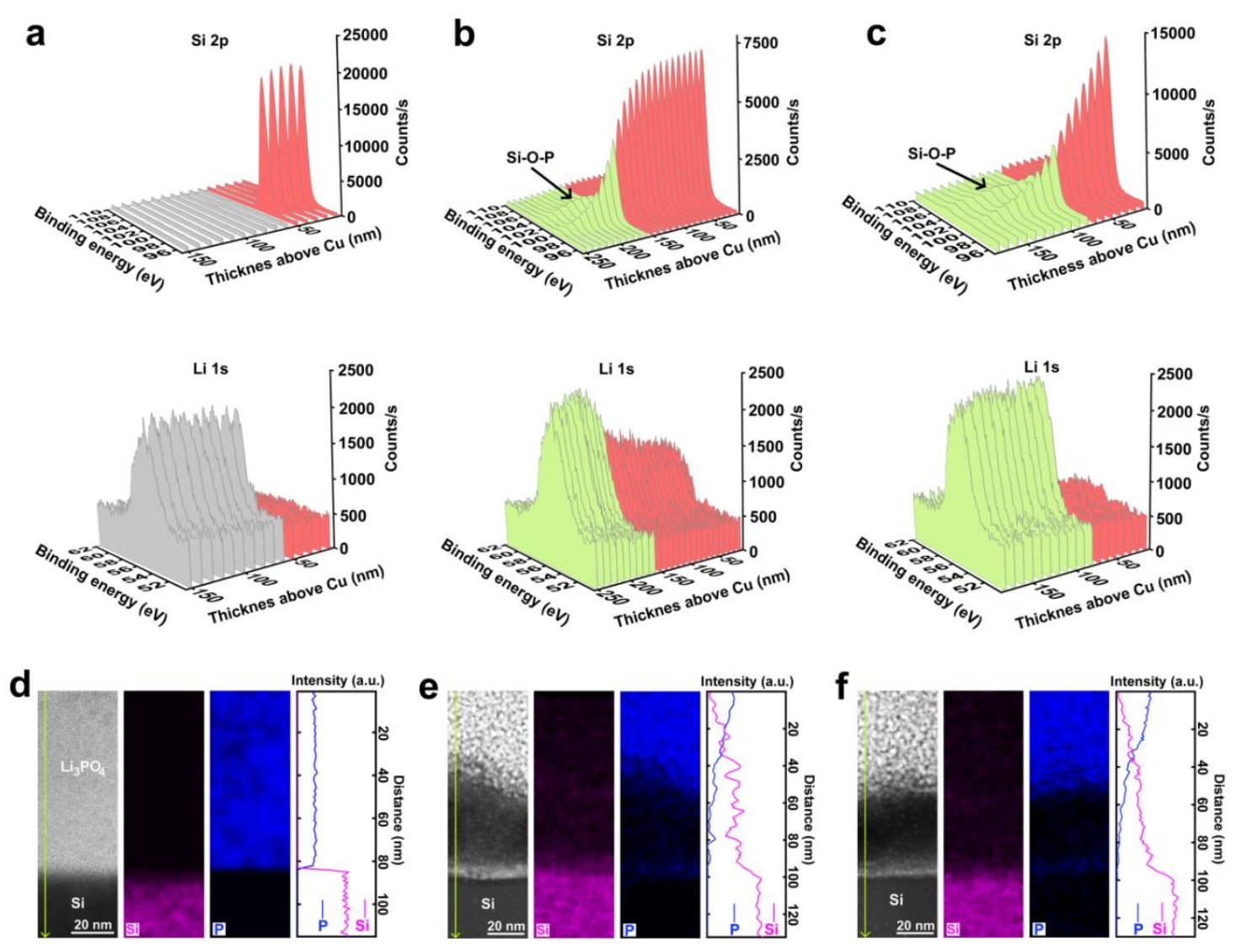

Figure 4. Three-dimensional spectra for $\mathrm{Si} 2 \mathrm{p}$ (first row) and $\mathrm{Li} 1 \mathrm{~s}$ (second row) as determined by XPS depth-profiling for a pristine (a), fully charged (b) and fully discharged all-solid-state battery (c). Red spectra represent the $\mathrm{Si}$ anode, the interfacial $\mathrm{Li}$-immobilization layer is identified by the green spectra whereas the grey spectra represent the pure $\mathrm{Li}_{3} \mathrm{PO}_{4}$ region. Cross-sectional HR-TEM image and corresponding EDX mapping of Si and $\mathrm{P}$, and EDX line scans across the $\mathrm{Si} / \mathrm{Li}_{3} \mathrm{PO}_{4}$ interface of a pristine (d), fully charged (e) and fully discharged battery (f). 
a
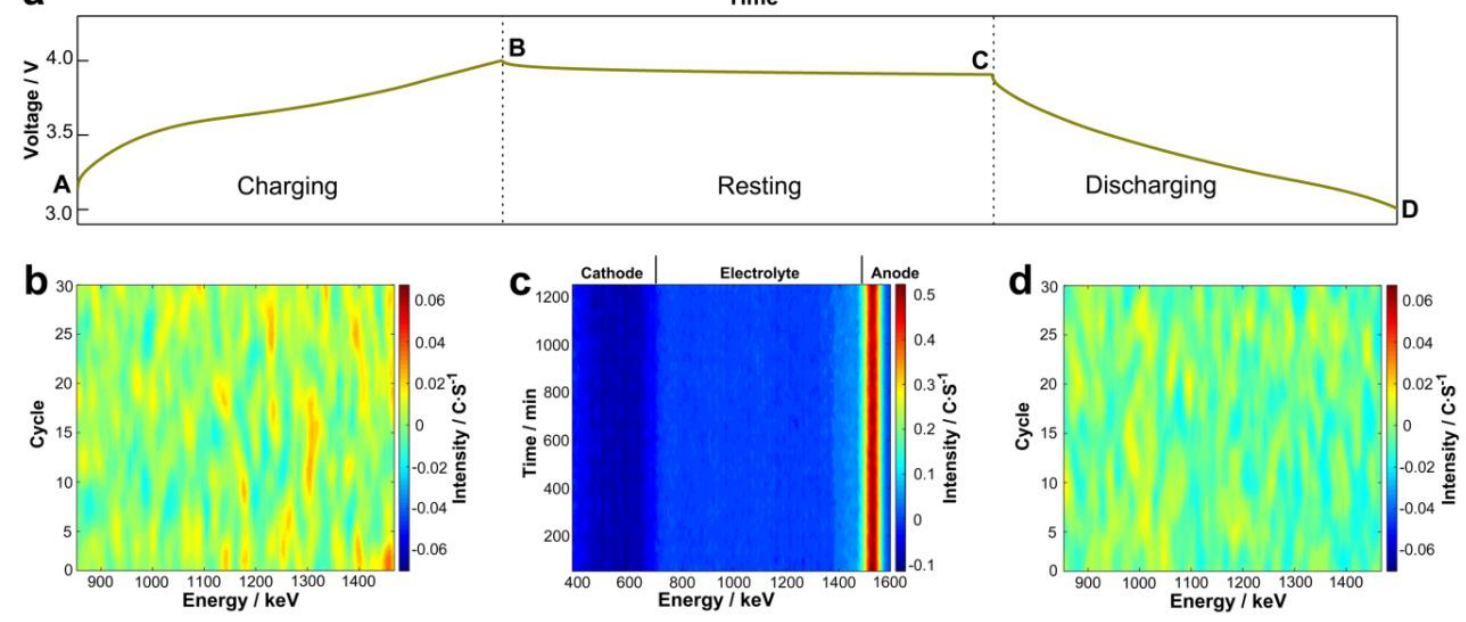

e
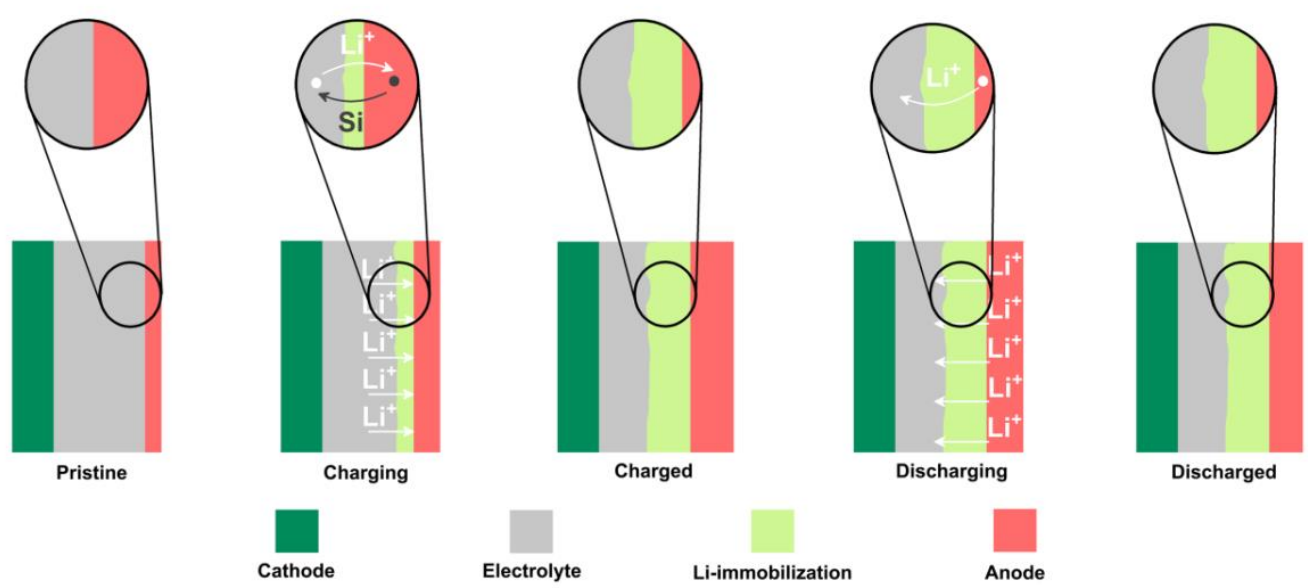

Discharging

Discharged

Figure 5. (a) Voltage charging, resting and discharging curve of a $\mathrm{Si}_{-}-\mathrm{Li}_{3} \mathrm{PO}_{4}-\mathrm{LiCoO}_{2}$ solidstate, thin film battery during which the NDP spectra has been continuously collected. (c) In operando spatial energy profiles during the 1260 minutes of resting after the first charging process. The increment spatial image for a fully charged (b) and fully discharged (d) battery during the first 30 cycles, of which the images were obtained by subtracting the spectra for each cycle before the charging (B-A) and discharging (D-C) processes has started, respectively. (e) Schematic representation of the evolution of the degradation mechanism of a $\mathrm{Si}-\mathrm{Li}_{3} \mathrm{PO}_{4}-\mathrm{LiCoO}_{2}$ thin film battery upon cycling, involving the formation of the $\mathrm{Li}-$ immobilization interlayer, originating from the $\mathrm{Si} / \mathrm{Li}_{3} \mathrm{PO}_{4}$ interface. 